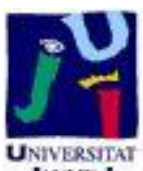

Título artículo / Títol article: Interplay Between Fullerene Surface Coverage and Contact Selectivity of Cathode Interfaces in Organic Solar Cells

Autores / Autors

Antonio Guerrero, Bernhard Dörling, Teresa Ripolles-Sanchis, Mahdieh Aghamohammadi, Esther Barrena, Mariano Campoy-Quiles and Germà Garcia-Belmonte

Revista:

ACS NANO, 7 (5) (2013)

Versión / Versió:

Cita bibliográfica / Cita bibliogràfica (ISO 690):
GUERRERO CASTILLEJO, Antonio... [et al.]. Interplay Between Fullerene Surface Coverage and Contact Selectivity of Cathode Interfaces in Organic Solar Cells. ACS NANO, 7 (5) (2013), p. 4637-4646.

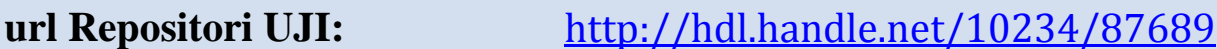




\title{
Interplay Between Fullerene Surface Coverage and Contact Selectivity of Cathode Interfaces in Organic Solar Cells
}

\author{
Antonio Guerrero ${ }^{1 *}$, Bernhard Dörling ${ }^{2}$, Teresa Ripolles-Sanchis ${ }^{1}$, Mahdieh
} Aghamohammadi ${ }^{2}$ Esther Barrena $^{2}$, Mariano Campoy-Quiles ${ }^{2}$ and Germà Garcia-Belmonte ${ }^{1 *}$

${ }^{1}$ Photovoltaic and Optoelectronic Devices Group, Departament de Física, UniversitatJaume I, ES-12071 Castelló, Spain

${ }^{2}$ Institut de Ciència de Materials de Barcelona (ICMAB-CSIC), Campus de la UAB, Bellaterra, 08193, Spain

*Corresponding author: A. Guerrero, email: aguerrer@uji.es and G. GarciaBelmonte, e-mail: garciag@fca.uji.es, tel.: +34 964 387548, fax: +34 964729218

\begin{abstract}
Interfaces play a determining role in establishing the degree of carrier selectivity at outer contacts in organic solar cells. Considering that the bulk heterojunction consists of a blend of electron donor and acceptor materials, the specific relative surface coverage at the electrode interfaces have an impact on the carrier selectivity. This work unravels how fullerene surface coverage at cathode contacts lies behind the carrier selectivity of the electrodes. A variety of techniques as variable-angle spectroscopic ellipsometry and capacitance-voltage measurements have been used to determine the degree of fullerene surface coverage in a set of PCPDTBT-based solar cells processed with different additives. A full screening from highly fullerene-rich to polymer-rich phases attaching the cathode interface has enabled the overall correlation between surface morphology (relative coverage) and device performance (operating parameters). The general validity of the measurements is further discussed in four donor/acceptor systems: PCPDTBT, P3HT, PCDTBT and PTB7 blended with fullerene derivatives. It is demonstrated that a fullerene rich interface at the cathode is a prerequisite to enhance contact selectivity, and consequently power conversion efficiency.

keywords: Organic Solar Cells, Metal-Organic Interfaces, Vertical Segregation, Surface Coverage, Contact Selectivity.

Publication: A. Guerrero et al. ACS Nano 7 (2013) 4637-4646.
\end{abstract}


Research on materials and processing conditions in bulk-heterojunction (BHJ) organic solar cells has led to impressive improvements over the last few years, with power conversion efficiencies (PCE) now reaching $10 \% .^{1}$ In a BHJ cell, an interpenetrating matrix of donor and acceptor molecules harvests sun light and generates charges via photoinduced charge transfer, that, finally are collected at selective electrodes. ${ }^{2}$ Film morphology of the donor:acceptor blends plays a crucial role in the overall device performance and has been widely studied. ${ }^{3}$ On the other hand, the interface between the organic active layer and the outer contact (including the surface morphology) also plays a very important role in the final device efficiency. Extensive work has been carried out to develop and optimize materials which allow for efficient extraction of charge carriers and enhance both power conversion efficiency (PCE) and cell stability. In order to obtain efficient selective contacts, two key requirements must be fulfilled: low contact resistance between the cathode or anode and the organic layer, ${ }^{4}$, 5 and adequate matching of energy levels to enhance electron or hole selectivity. ${ }^{6}$ Several materials are available to enhance the electron extraction selectivity at the cathode contact, including alkali metal compounds $(\mathrm{Ca}, \mathrm{LiF} \ldots)$, metal oxides $\left(\mathrm{TiO}_{x}\right.$, $\mathrm{ZnO} \ldots$ ), and low-molecular weight organic materials. ${ }^{7-9}$ Regarding the extraction of holes at the anode, the most commonly used materials are poly $(3,4-$ ethylenedioxythiophene):poly (styrenesulfonate) (PEDOT:PSS) ${ }^{10}$ and $\mathrm{MoO}_{3} \cdot{ }^{11,12}$ These additional layers have been clearly proven as useful contact selectivity enhancers. However, when considering that the bulk heterojunction consists of a blend of electron donor and acceptor materials, the specific relative surface coverage at the electrode interfaces is likely to also have an impact on the carrier selectivity. It is recognized that the nanoscale-size blend structure attaching the contact is a much less explored terrain, and therefore constitutes the main aim of this work.

Currently, a wide range of physical techniques is available to provide information on the properties of the blends and interfaces. ${ }^{13,6}$ Techniques such as Grazing Incidence Xray Diffraction (GXID), Atomic Force Microscopy (AFM) or Transmission Electron Microscopy (TEM) have been generally used to characterize the structure and length scales of the donor:acceptor domains. Additionally, information related to the depth profile concentration of donor:acceptor blends has been obtained by means of Grazing Incidence X-Ray Diffraction (GIXRD), ${ }^{14}$ neutron reflectivity, ${ }^{15}$ Near-Edge X-ray Absorption Fine Structure Spectroscopy (NEXAFS), ${ }^{16}$ Dynamic secondary ion mass 
spectroscopy (SIMS), ${ }^{17}$ Variable-Angle Spectroscopic Ellipsometry (VASE), ${ }^{18}$ etc. Using this last technique, both vertical and lateral distribution of the individual components has been reported on blend films.

These techniques can be extremely useful to characterize the film morphology. They have, however, some practical limitations when a direct comparison between structure and device performance is desired to extract meaningful correlations for solar cell operation. On the one hand, some of them are destructive (cross-section SEM or SIMS), and on the other, accuracy is compromised when the metallic top contact is deposited (e.g. VASE). In particular, attempts to obtain a direct relationship between vertical phase segregation and contact selectivity in operating cells have provided unexpected contradictory results. For example, using NEXAFS a morphology comprising three different phases has been suggested for the P3HT:PC ${ }_{61} \mathrm{BM}$ system, ${ }^{19}$ in which a blended phase is sandwiched between two thin P3HT layers at both anode and cathode interfaces (see Figure 1a for a schematic representation). A P3HT upper layer, adjacent to the cathode is expected to be detrimental to device performance due to the $p$-character of the polymer; however, the observed good performance has been justified by the diffusion of aluminum during deposition or the annealing step. Importantly, the specific processing conditions employed to fabricate the samples for a structural study may differ from those adapted for device fabrication, which results in different composition depth profiles, making comparisons unreliable. Therefore, a technique that could provide structural information at the contact/active layer interface on working cells would contribute to establish a direct relationship between vertical segregation (e.g. donor/acceptor coverage at the interface) and device performance (e.g. contact selectivity) and could potentially help to disentangle the respective contributions to the cell operation resulting from surface coverage by organic compounds and extracting/blocking interlayers.

Recently, we have used capacitance-voltage $(C-V)$ measurements to understand the underlying mechanisms involved in the formation of dipoles at the active layer/cathode contact interface. ${ }^{20}$ We have proposed a simple, albeit robust, model based on the role of the charge-neutrality level (CNL) located at a given position within the interfacial density-of-states (IDOS). CNL represents a key parameter accounting for the interface energy equilibration. ${ }^{21-23}$ In this previous work we noticed that $C$ - $V$ measurements are very sensitive to all interfacial materials: donor, acceptor, metallic contact, and their 
relative concentration. Regarding the donor and acceptor molecules, not only was important the position of the HOMO and LUMO levels, but also the ratio of each of the components at the interface. It was observed that the only way to assure efficient electric contact between the cathode metal and the active layer bulk is the presence of chemically reduced fullerene molecules attached to the interface. ${ }^{20}$

The specific aim of this work is to correlate fullerene surface coverage and BHJ solar cell performance by evaluating cathode carrier selectivity. To this end we use ellipsometry and a purely electrical technique $(C-V)$ that in combination provide information regarding the relative donor/acceptor surface coverage at the active layer/cathode interface in operating devices. For this purpose, we have chosen the low band gap polymer poly[2,6-(4,4-bis-(2-ethylhexyl)-4H-cyclopenta [2,1- $\left.b ; 3,4-b^{\prime}\right]$ dithiophene)-alt-4,7(2,1,3-benzothiadiazole)] (PCPDTBT) and the soluble fullerene (6,6)-phenyl $\mathrm{C}_{71}$ butyric acid methyl ester $\left(\mathrm{PC}_{71} \mathrm{BM}\right)$, and fabricated films with processing additives: namely hexanedithiol (HDT), octanethiol (OT) and octanedithiol (ODT). This allowed us to create a case study for investigating the vertical segregation. To get insight on the properties of the blend/anode system before cathode deposition, Kelvin Probe and Kelvin Probe Force Microscopy are employed. Then, we compare the $C-V$ results in completed cells and the vertical profiles deduced with a well established technique as spectroscopic ellipsometry. The good agreement obtained between these techniques confirms the validity of $C-V$ for deducing surface coverage ratios. Moreover, we investigate the generality of the technique by testing three different donor/acceptor systems. The donors under study poly(3-hexylthiophene) (P3HT), poly[[9-(1octylnonyl)-9H-carbazole-2,7-diyl]-2,5-thiophenediyl-2,1,3-benzothiadiazole -4,7-diyl2,5-thiophenediyl] (PCDTBT) and poly(\{4,8-bis[(2-ethylhexyl)oxy]benzo[1,2-b:4,5$\mathrm{b}^{\prime}$ ]dithiophene-2,6-diyl $\}$ \{3-fluoro-2-[(2-ethylhexyl)carbonyl]thieno[3,4- $b$ ]

thiophenediyl $\}$ ) (PTB7) were used either with $(6,6)$-phenyl $\mathrm{C}_{61}$ butyric acid methyl ester $\left(\mathrm{PCB}_{61} \mathrm{M}\right)$ or $\mathrm{PC}_{71} \mathrm{BM}$ to provide the following systems: P3HT:PCB ${ }_{61} \mathrm{M}$, PCDTBT:PC ${ }_{71} \mathrm{BM}$ and PTB7:PC 71 BM. We finally found that solar cell parameters as leakage current, short-circuit current, and fill-factor are highly determined by the degree of fullerene surface coverage at the cathode interface, which can be controlled by using proper processing additives.

\section{Results and Discussion}

Vertical segregation measured by variable-angle spectroscopic ellipsometry 


\section{measurements}

We start by characterizing the composition profile across the depth of films using ellipsometry. This highly sensitive and non-destructive spectroscopic technique has been successfully employed by us ${ }^{18,14,24}$ and other groups ${ }^{25-26}$ to characterize vertical segregation in bulk heterojunction blends. Here we will study thin blends ( $c a .40 \mathrm{~nm})$ of the system PCPDTBT:PC ${ }_{71} \mathrm{BM}$ prepared using different additives as discussed in the methods section. Before modeling the blends, we have determined the refractive index $(n)$ and extinction coefficient $(\kappa)$ of the specific materials that we have employed, deposited using the protocol described in the experimental. For the substrate materials (ITO and PEDOT:PSS) the dielectric function was modeled using a Lorentz oscillator assembly coupled to a Drude tail at low frequencies. For PCPDTBT and $\mathrm{PC}_{71} \mathrm{BM}$, we employed the Standard Critical Point $\operatorname{model}^{27}$ of $n$ and $\kappa$. These data are shown in Supporting Information (Figure SI2). Importantly, the absorption bands of the polymer do not change significantly upon blending (Figure SI1). This implies that the reference $n$ and $\kappa$ of neat materials can be used to characterize the blends by means of effective medium approximations (Bruggeman). In accordance with the previous comments AFM images in Figure SI5 evidence the small degree of phase separation at the surface of the analyzed layers.

We have used a range of models to fit the ellipsometric experimental data for the blend films on ITO/PEDOT:PSS substrates. Reassuringly, the deduced profiles are consistent for the different models (see Figure SI3). This consists of a fullerene rich sublayer in the middle of the film depth, sandwiched between two polymer rich layers at the substrate and air interfaces, as schematically illustrated in Figures 1a and $1 \mathrm{~b}$. Incorporating additives in the blend solution quantitatively modifies the profile, while keeping the general trend the same. Interestingly, the deduced $\mathrm{PC}_{71} \mathrm{BM}$ coverage at the surface and the depth of the surface sub-layer increases when going from the sample processed without additives to those with additives. The volume concentration of $\mathrm{PC}_{71} \mathrm{BM}$ at the surface is highest for ODT, then for OT, then HT and finally for the noadditives sample. The full profile suggest that the fullerene molecules enriching the surface result from the depletion of $\mathrm{PC}_{71} \mathrm{BM}$ from the substrate side of the film, which becomes more PCPDTBT rich. These results will be compared to $C$ - $V$ measurements in following sections. 
a)

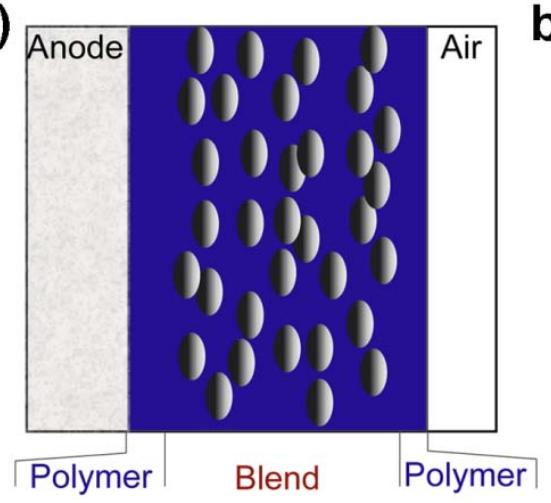

b)

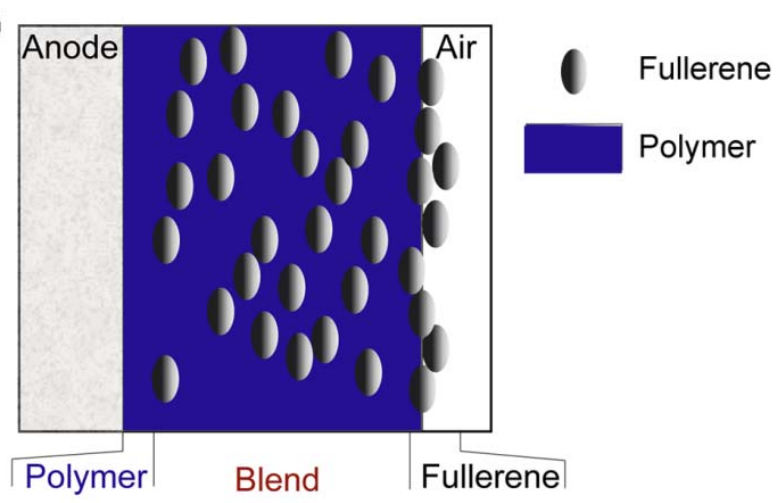

c)

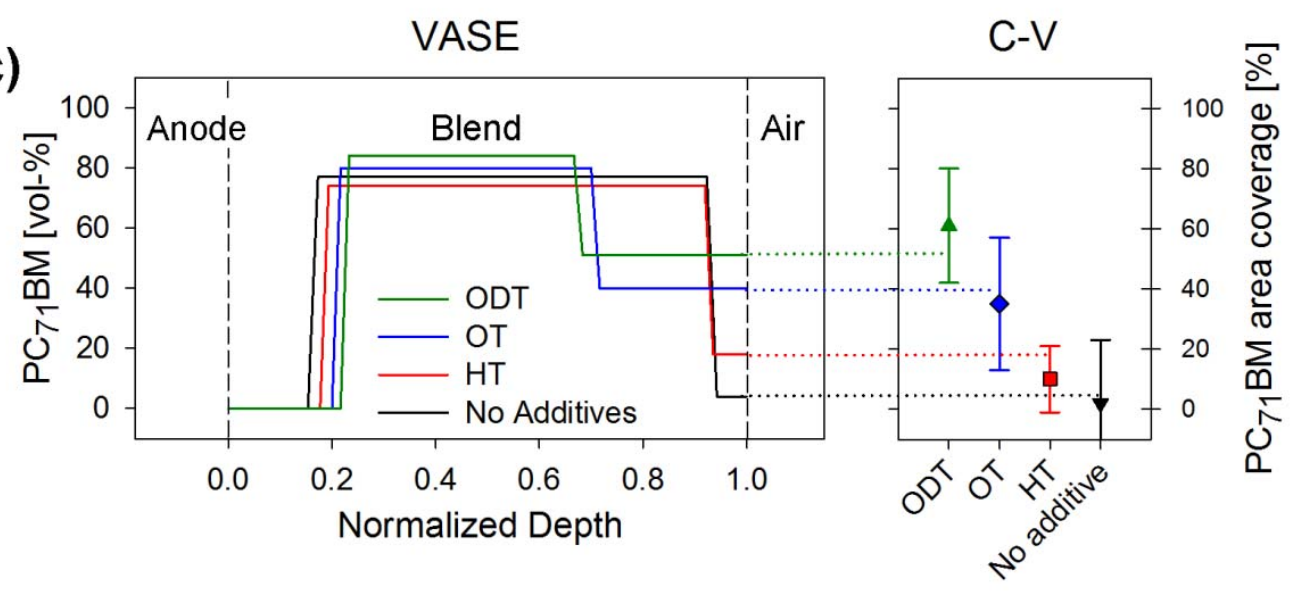

Figure 1: Schematic illustration to show: (A) Segregation based in three phases with the configuration polymer/blend/polymer, (B) Ideal phase segregation with the configuration polymer/blend/fullerene. (C) Comparison of phase segregation profile as inferred from VASE with fullerene surface concentration obtained by capacitancevoltage technique.

\section{Surface potential of anode/active layer films}

VASE strongly indicates a dependence of acceptor/donor relative surface coverage on the specific additives used in the processing of the films. We set now to investigate by Kelvin Probe (KP) measurements the surface potential of the anode/active layer film system before the top cathode deposition. KP technique nullifies the surface potential difference between two surfaces brought in close proximity (flat-band conditions), which in the case of two metals corresponds to the difference of their respective work functions. In a complex system such as a polymer:fullerene film deposited on a metal electrode, the measured surface potential represents an effective, average work function $\phi_{\text {org }}$. This contains information of the interfacial dipoles or band bending that may 
originate from the respective energy alignment of both materials and the anode. It should be noted that other factors such as unexpected trap charges at the surface of the organic $^{28,29}$ or variations in crystallinity and orientation of the polymer may be reflected in the observed surface potential, and the interpretation is not always straightforward.

$\mathrm{KP}$ measurements were first performed with a $2 \mathrm{~mm}$ wide tip which is representative for devices as it provides a relatively large area. Measurements were carried out in the dark and in nitrogen atmosphere by using a configuration ITO/PEDOT:PSS/organic layer. WF values were calculated by calibrating the tip with a gold sample. Figure 2a shows WF values of pure films of PCPDTBT and $\mathrm{PC}_{71} \mathrm{BM}$ and blends processed with and without ODT additive. Low values are found for the sample containing the pure polymer $(-4.7 \mathrm{eV})$ and high values for the pure fullerene $(-5.1 \mathrm{eV})$. As observed in Figure 2a, WF encountered for fullerene film lies at midgap values. This informs on the negligible doping level of acceptor molecules as expected for chemically stable fullerenes. However, polymer films exhibit $p$-doping character to some extent as inferred from the WF position displacement approaching the donor HOMO level. On the other hand, intermediate values are obtained for blends processed with and without additives and these only differ in about $\sim 70 \mathrm{mV}$. This downward shift with respect to the polymer WF can be understood as a double effect of $\mathrm{PC}_{71} \mathrm{BM}$ molecules by increasing the polymer doping and averaging the blend WF. This result is a clear indication that the organic/anode interface is not largely affected by the introduction of additives and processing conditions.

Additionally, in order to understand whether we are able to observe different results at the nanoscale Kelvin Probe Force Microscopy (KPFM) has been employed to map the WF of the films with larger spatial resolution. Figure 2 shows topography (c and $f$ ), resonance frequency shift ( $\mathrm{d}$ and $\mathrm{g}$ ), and contact potential maps (e and $\mathrm{h}$ ) for blends without (top) and with (bottom) ODT. Whereas topography images are dominated by surface roughness, the frequency shift is more sensitive to the nanostructure of the organic films. Interestingly, the images show a remarkable resemblance between the morphology and the contact potential maps in the two types of samples with RMS roughness (as deduced from $10 \times 10 \mu \mathrm{m}^{2}$ areas, not shown) being $0.55 \mathrm{~nm}$ and $0.46 \mathrm{~nm}$, respectively. This seemingly lack of differences in the surface morphology and contact potential maps is also apparent when looking at other superficial traits, such as adhesion (see Supporting Information), and evidences a fine nanoscale intermixing of the two 
components constituting this system. ${ }^{30}$ The averaged $\phi_{\text {org }}$ obtained from surface potential images is very similar regardless of the use of ODT (Supporting Information). Regarding the calculated WF values these are very similar to those obtained by KP and the small differences may be ascribed to the fact that films were exposed to air before the KPFM measurements.

Overall, the similarity of surface potential for both blends measured by KP and KPFM is a strong indication that the organic/anode interface is not largely affected by additive processing in regards to its electronic properties. This agrees well with the results from VASE that show a polymer rich layer at the anode interface. Although the observed trends by VASE concerning fullerene coverage on the top active layer/air surface are qualitatively reproduced by Kelvin probe analyses, the surface voltage variation amounts only $\sim 70 \mathrm{mV}$ for the two blends studied. As explained below large differences are found regarding the additive effect on solar cell performance. This observation point to the fact that electronic equilibration after cathode interface formation, and the consequent dipole contribution, is a key mechanism to understand the photovoltaic performance. This issue is addressed by measuring $C-V$ characteristics. 


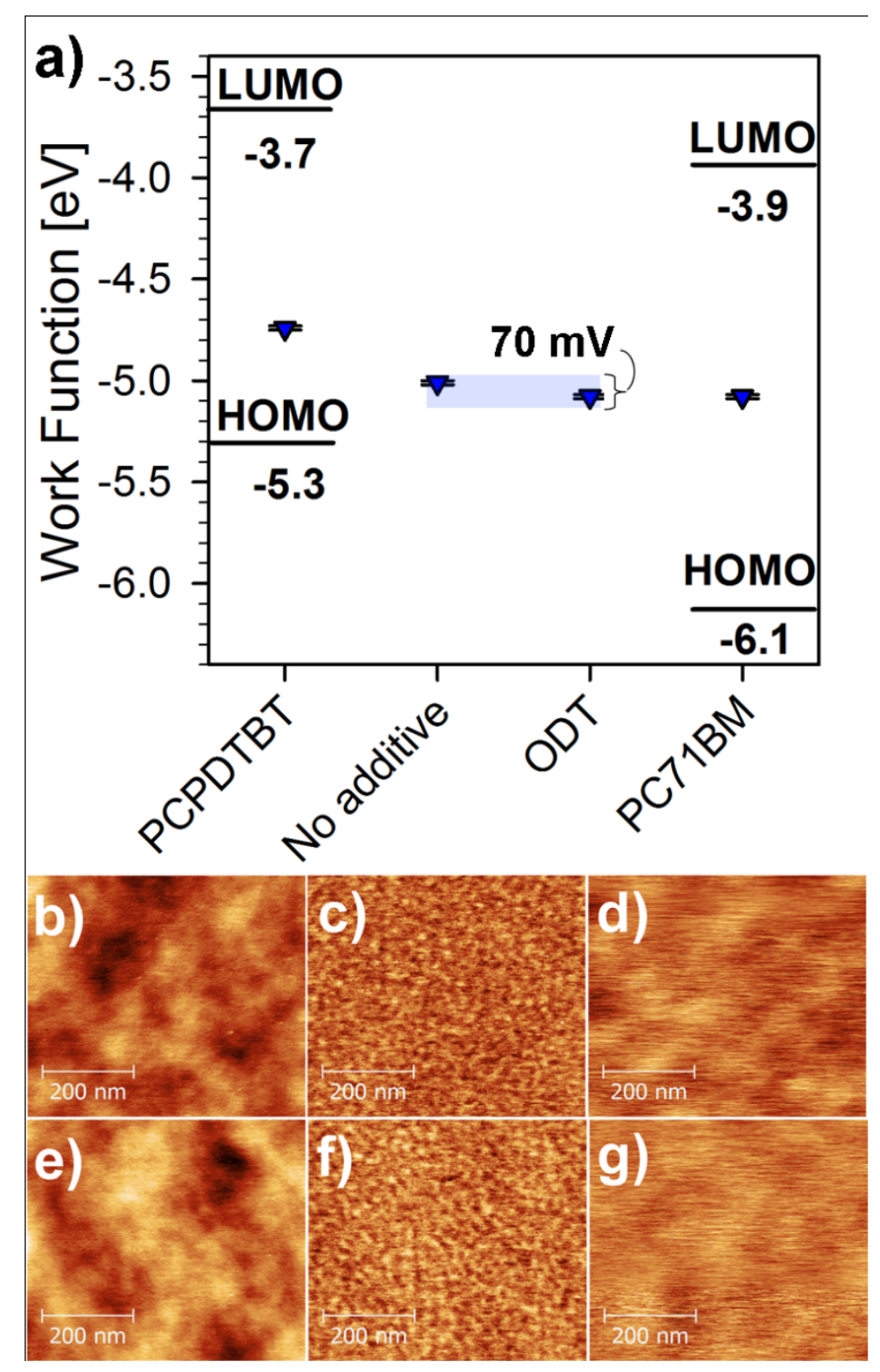

Figure 2: (A) Work function values of the ITO/PEDOT:PSS/organic films measured by KP. Topography (B and E), frequency shift (C and F) and surface potential (D and G) for the ITO/PEDOT:PSS/ PCPDTBT:PCBM blends without ODT (top) and with ODT additive (bottom).

\section{Fullerene surface coverage quantified by capacitance-voltage measurements}

VASE strongly indicates both a vertical segregation of components and its dependence on the processing with additives. These characterizations have been carried out on films in the absence of the metallic cathode. Although samples containing an ultrathin semitransparent electrodes could be explored using VASE, ${ }^{18}$ a more reliable comparison between surface coverage and device performance would be obtained if 
complete and operational devices are tested. In particular, correlation between interfacial composition and contact selectivity could be of great importance to understand the role that vertical segregation plays in device performance.

We have recently reported an extensive investigation on the cathode energy equilibration of organic bulk-heterojunction solar cells using the information extracted from an exclusively electrical technique as $C$-V analysis. ${ }^{20}$ Our aim here is to investigate whether $C-V$ measurement can be confronted with the previous experiments in order to evaluate mechanisms governing the interface energy level alignment. Of particular interest is to quantifying the relative surface coverage at the active layer/cathode interface in operating solar cells. ${ }^{20} \mathrm{We}$ showed that the applied potential required to balance the mismatch between the cathode metal and the organic layer Fermi levels is divided into two contributions: a spatially extended band bending in the organic bulk, and a voltage drop at the interface dipole layer which is caused by a net charge transfer between the cathode metal and the organic blend. ${ }^{20}$

Figure 3 a represents schematically the situation before the contact between the active layer and the metal cathode is made. The relevant energy levels of the separated parts are shown. On the active layer side it is known that unintended oxidation of the donor polymer occurs during processing by the presence of oxygen or humidity as previously shown for P3HT: $\mathrm{PC}_{60} \mathrm{BM}^{31}$ This confers $p$-doped character to the polymer yielding as a result a background concentration of mobile holes. The position of the Fermi level is then shifted down closer to the HOMO level of the donor molecules compared to that observed for undoped blends. The polymer doping is corroborated here by the measured WF position as explained previously when KP results were shown. Once the active layer is contacted to the cathode, the energy equilibration entails the Fermi level alignment with a concomitant vacuum level offset that is divided into two contributions: a) band bending inside the active layer which makes up a depletion zone in the vicinity of the cathode, and $b$ ) an interface dipole layer which accommodates part of the vacuum level offset (Figure 3b). ${ }^{32}$ The dipole layer appears as a consequence of a negative charge that is injected on the organic side reducing adjacent fullerene molecules. This negative charge is compensated by a positive charged layer at the metal contact.A simple model that integrates both voltage drops into a unified description of the contact equilibration was recently proposed. ${ }^{20}$ An expression for the cathode equilibration can be written as follows 


$$
q V_{\mathrm{fb}}=E_{F}-\phi_{\mathrm{c}}-\Delta
$$

where $q V_{\mathrm{fb}}$ is the voltage required to overcome the active layer band bending that leads to flat band condition, $E_{F}$ is the Fermi level of the active layer (interpreted in terms of the bulk work function), $\phi_{\mathrm{c}}$ is the metal Fermi level, and $\Delta$ is an interfacial dipole generated between active layer and cathode contact.

A key parameter accounting for the interface equilibration is the charge-neutrality level (CNL), which is defined as the energy level that results in a surface without net charge. ${ }^{21-23}$ When the chemical interaction between the metal and contacting conjugated molecules or polymers is not negligible, it is expected that molecules attached to the metal surface undergo both a shift and a broadening of their molecular energy levels. Energy distribution of the attached molecules should be modeled by a specific interfacial density of states (IDOS) which differs from that encountered in the bulk of the organic layer. CNL is located at a given position within the interfacial density of states (IDOS) appearing because of the highly disordered energy landscape. We observed that the strength of the flat band potential depends on the polymer-to-fullerene ratio at the surface. ${ }^{20}$ 


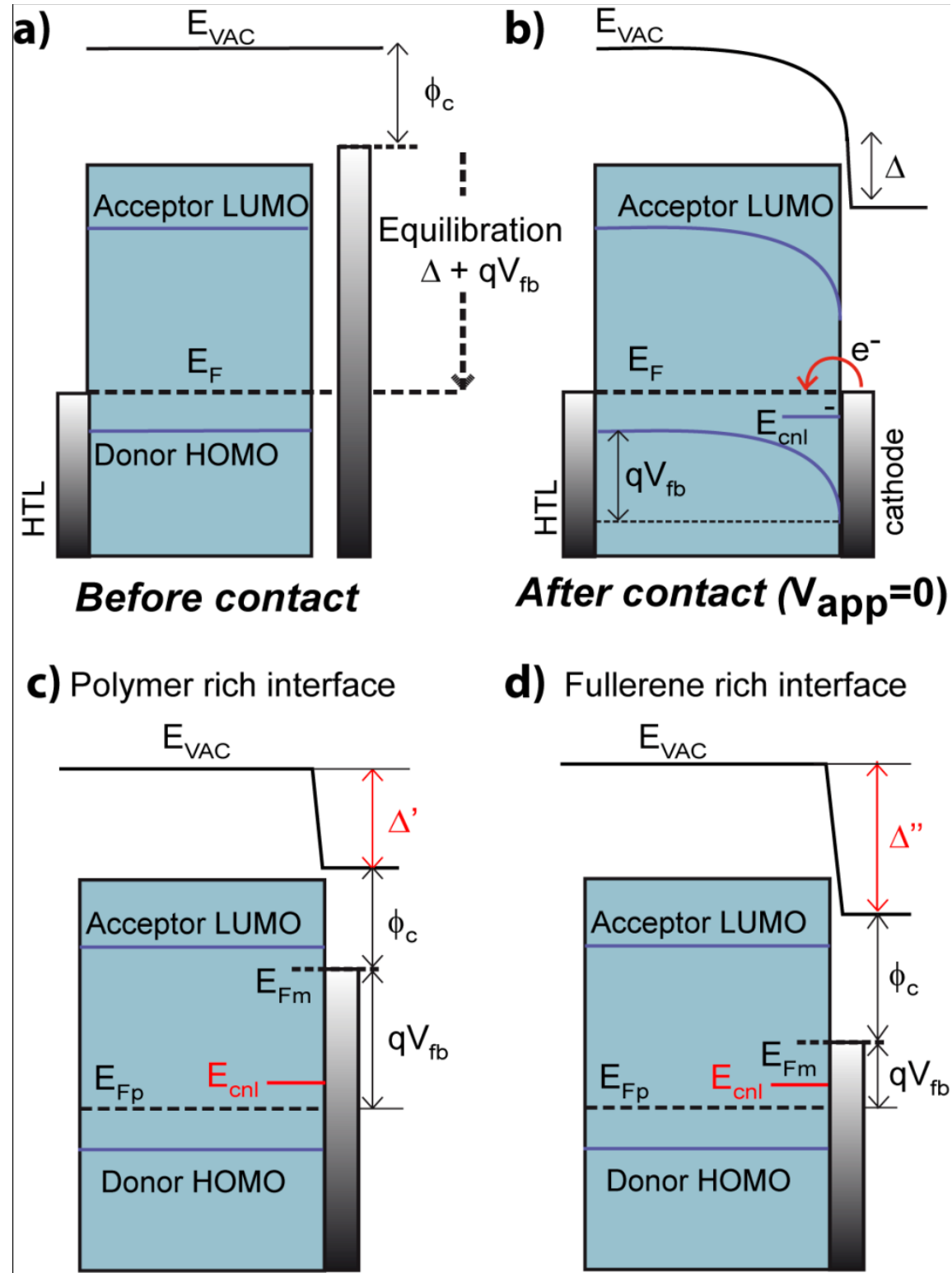

Flat-band conditions $\left(\mathrm{V}_{\mathrm{app}}=\mathrm{V}_{\mathrm{fb}}\right)$

Figure 3: Energy level diagrams representing the cathode equilibration of a bulkheterojunction solar cell (A) before contact equilibration, (B) after contact deposition, (C and D) effect of CNL on dipole generation induced by donor/acceptor concentration at the active layer/cathode interface. $E_{F m}$ corresponds to the metal Femi level, $E_{F}$ is the equilibrium Fermi level of the active layer, $E_{\mathrm{cnl}}$ is the CNL energy position. It is assumed that out of equilibrium $E_{F p} \approx E_{F}$. Other energy differences correspond to that explained in eq 1 .

$C-V$ response is shown in Figure $4 \mathrm{a}$ for devices prepared with pure polymer 
PCPDTBT, a fullerene-rich blend (polymer:fullerene ratio of 1:6) and two selected devices fabricated from blends (1:2 ratio) without additive and with ODT. In the series of films under study, the capacitance response at intermediate frequencies $(100 \mathrm{~Hz}-1$ $\mathrm{kHz}$ ) shows the expected plateau in reverse and low-forward voltage consequence of full depletion conditions. At a certain forward voltage, the capacitance starts to increase consequence of the depletion width reduction. For this voltage range the Mott-Schottky (MS) analysis can be carried out (Figure $4 \mathrm{a}$, right graph). ${ }^{31}$ In $C^{-2}(V)$ plot, $V_{\mathrm{fb}}$ corresponds to the intercept with the voltage-axis. High $V_{\mathrm{fb}}$ values are obtained for the device fabricated with PCPDTBT polymer only. This is due to the fact that the dipole contribution plays a secondary role, being the work function mismatch between polymer and metal mainly accommodated by the bulk band bending (Figure 3c). On the other hand, high concentrations of fullerenes in the blend (polymer:fullerene ratio of 1:6) lead to stronger dipoles because of the larger charge associated with fullerene reduction reaction (charge transfer from the metal). The overall net effect is the practical alignment of the metal Fermi level $\left(E_{F m}\right)$ and CNL position (Figure 3d). Under such as interface equilibration flat-band voltage is reached at much lower values. Implicitly the model assumes that no band bending is taking place during equilibration at the anode due to a correct energy level alignment of the polymer HOMO level with the PEDOT:PSS. Note that values of $\phi_{\text {org }}$ for blends with/without ODT measured by KP only differ about $70 \mathrm{mV}$ in strong contrast with the difference of above $0.7 \mathrm{~V}$ in $V_{\mathrm{fb}}$ values obtained from $C$ - $V$ analysis (Figure $4 \mathrm{~b}$ ). Importantly we found differences of more than $1 \mathrm{~V}$ in $V_{\mathrm{fb}}$ among the set of analyzed devices. There is a significant variation in the dipole layer strength depending on the additive and processing conditions. We highlight here that this last observation is in agreement with results obtained by VASE. 


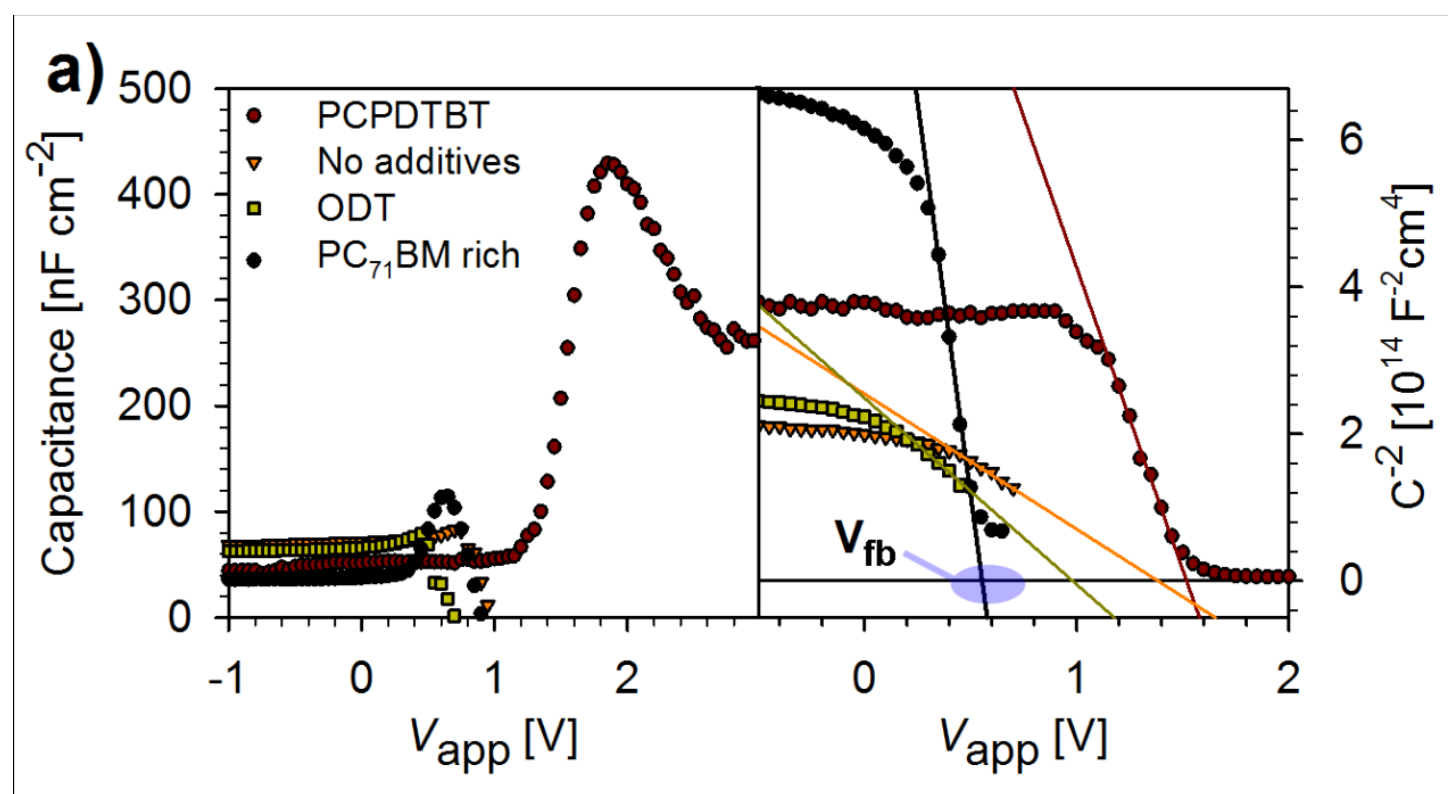

b)

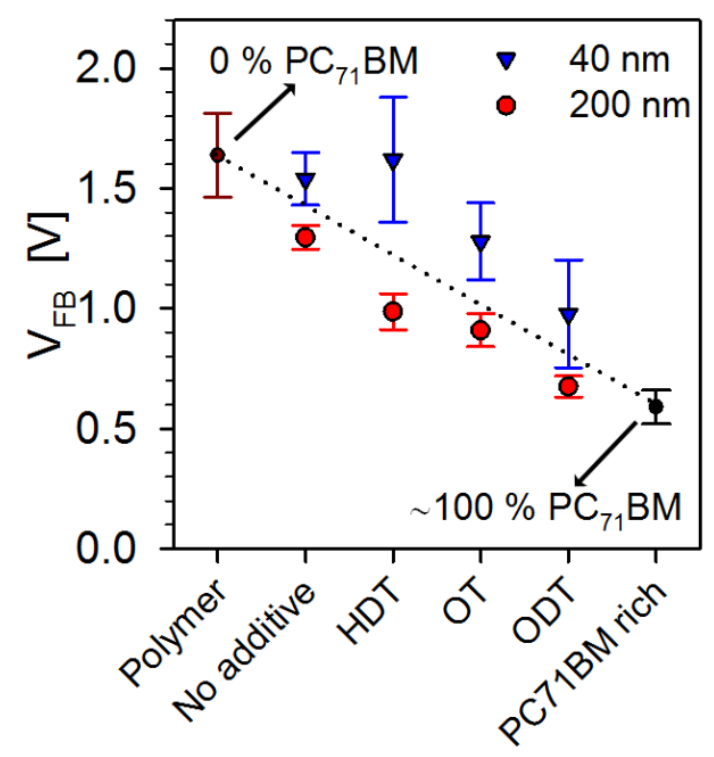

Figure 4: (A) Capacitance-voltage response (left) and Mott-Schottky analysis (right) of a selection of representative devices processed under different conditions. Data values influenced by the decrease in capacitance have been removed in MS plot for clarity. The $V_{\mathrm{fb}}$ is graphically estimated from the intercept with the x-axis. (B) $V_{\mathrm{fb}}$ results extracted from $C-V$ measurements in the dark for devices processed under different conditions. Two sets of devices with different active layer thickness are included that show that the method is valid for different active layer thicknesses.

Figure $4 \mathrm{~b}$ shows values of $V_{\mathrm{fb}}$ for a whole series of devices fabricated using different additive conditions. $V_{\mathrm{fb}}$ of a device fabricated from a blend with no additive is slightly 
lower than that encountered the only polymer films. When ODT is used the value approaches that of the fullerene rich device. Large dispersion of results averaging over 5 devices is observed for thin films $(40 \mathrm{~nm})$ possibly due to the large impact of metal diffusion for this low device thickness. All in all it is important to note that the cathode of these devices was fabricated at slow evaporation rates $(0.2-0.8 \AA / \mathrm{s})$. Cells based on thicker films are considerably less sensitive to diffusion of the cathode metal and results show a standard deviation of about $5 \%$. Not surprisingly $V_{\mathrm{fb}}$ values for the thick cells differ from those obtained for the thin cells as a result of the expected different phase segregation and band bending profile.

Because of the high sensitivity of $V_{\mathrm{fb}}$ on blend structure and additives used, we adopt it as a measurement of the fullerene coverage of the contact surface. We can take $V_{\mathrm{fb}}$ of pure PCPDTBT and fullerene-rich devices to account for the extreme cases of full active layer/cathode coverage by polymer and fullerene, respectively. If it is assumed that a linear relationship between $V_{\mathrm{fb}}$ values and surface coverage occurs, the $V_{\mathrm{fb}}$ results of devices fabricated with additives can be employed to estimate the relative surface coverage percentage. We note here that the capacitance analysis is sensitive to the features of the fullerene IDOS. Figure 1 shows that the fullerene surface coverage percentage for the series correlates well with results obtained by VASE. This good agreement clearly indicates that $C$ - $V$ measurement is a useful tool to detect the degree to which fullerene or polymer is present at the active layer/cathode interface in complete devices. It is important to remark here that some discrepancies between metal free samples and completed cells can be expected, as metal diffusion and surface morphology modification during evaporation are expected to occur. In addition, Figure $4 \mathrm{~b}$ clearly shows that $V_{\mathrm{fb}}$ obtained for the $200 \mathrm{~nm}$ active layer thickness devices are lower than values obtained for the thin device analogues. Then, following the same analysis the fullerene active layer/cathode surface coverage is slightly higher for the devices with a thick active layer. In the same line, optimized devices which are typically fabricated with an active layer of $80-100 \mathrm{~nm}$ should show intermediate fullerene coverage to those presented in this work. In any case the same trend is encountered regardless the film thickness. Moreover, as we will discuss in the last section, the degree to which the cathode is covered by polymer plays a significant role in the device performance parameters due to variation in contact selectivity. 
In order to study whether $C-V$ analysis can be used generally to measure the fullerene/polymer concentration at the active layer cathode interface, we carried out a similar analysis in a range of different donor:acceptor systems. In particular, we focus on the donors polythiophene P3HT:PC ${ }_{61} \mathrm{BM},{ }^{33}$ polycarbazole $\mathrm{PCDTBT}: \mathrm{PC}_{71} \mathrm{BM}^{34-36}$ and high efficiency PTB7:PC ${ }_{71} \mathrm{BM}^{37}$ Besides resulting in higher power conversion efficiencies, these materials vary in their tendencies to self-aligned (from highly crystallizable to almost glassy), as well as in the protocols that have been identified in order to control their morphology (thermal annealing and additives), and thus in combination with PCPDTBT: $\mathrm{PC}_{71} \mathrm{BM}$ provide a good span of material properties and processing schemes to generalize our findings. A complete analysis is provided as Supporting Information, signaling that $C-V$ technique is also sensitive to surface coverage for these systems (Figure SI6) and analogous analysis to measure the fullerene area coverage can be carried out.

\section{Correlation between surface coverage and device performance}

In order to design bulk-heterojunction solar cells with optimum efficiencies, apart from a perfect control of film morphology, engineering of interfacial layers is of vital importance. This is so because strongly selective electrodes will minimize leakage current which is an indication of undesired hole movement crossing the cathode contact. Although detailed discussion on the relation of phase segregation with the overall cell performance is complex, we will discuss in detail our results for the case study PCPDTBT:PC ${ }_{71} \mathrm{BM}$ system and general conclusions are partially extrapolated for other blend combinations such as those shown in the Supporting Information.

AFM analysis previously discussed shows a lack of surface morphology differences between samples processed with additives from those not using additive. This is in contrast to the case of $\mathrm{P} 3 \mathrm{HT}: \mathrm{PC}_{61} \mathrm{BM}$ system, for which micron-scale $\mathrm{PC}_{61} \mathrm{BM}$ aggregates appear protruding from the surface upon annealing. ${ }^{18}$ The apparent lack of information regarding different surface coverage resulting from SPM data, readily accessible using VASE and $C-V$, is likely due to the nanoscale intermixing of the two components constituting this blend, ${ }^{30}$ and warns that vertical segregation in highly mixed blends cannot be accessed using surface microscopy. Additionally, it is important to note that the morphological information extracted in our measurements differ to those results previously reported by Peet et al.. ${ }^{30}$ In their AFM measurements these authors 
were able to observe enhanced degree of phase separation in the blend induced by the high boiling point of the ODT additive and the preferential solubility of the fullerene in the additive compared to the polymer. Therefore, it appears that under our processing conditions in which we obtain extremely thin active layers (i.e. $40 \mathrm{~nm}$ ) the scenario regarding film morphology is rather different. Here no large differences in phase separation between samples treated with additives with those untreated are observed, and absorption suggests similar degree of crystallinity. This observation reinforces the hypothesis that the interface is playing a determining role and explains difference in device performance.

Performance parameter results of devices are shown in Table 1. The use of the additives with different boiling points in the case of PCPDTBT:PC ${ }_{71} \mathrm{BM}$ clearly impacts the final device performance. All device performance parameters (power conversion efficiency, open-circuit voltage $V_{\text {oc }}$, short-circuit current $J_{\text {sc }}$ and $F F$ ) are found to be maximum when ODT is used and these gradually decrease for devices fabricated without additives (see Table 1 and Figure 5a). Diode response of the same devices in the dark (Figure 5b) clearly show a trend in the measured current at $-1 \mathrm{~V}$ (leakage current): high currents for devices containing low proportion of fullerene at the cathode and low leakage currents for the best performing device. The same results are found for the calculated shunt resistances $R_{\mathrm{sh}}$ that are about one order of magnitude higher for the device that contain a low proportion of fullerene at the contact. Injection of holes from the cathode contact at reverse bias originates then low shunt resistances. Both facts are a clear manifestation of enhanced selectivity of the contact when a high proportion of fullerene is present at the cathode.

Moreover, when the solar cell functions in the power-generating quadrant (forward voltage under illumination) the polymer-rich surface in the vicinity of the cathode offers an additional resistance to the collection of electrons. Accordingly, the total series resistance of the device increases because of the presence of the polymer layer, strongly affecting both $F F$ and achieved photocurrent. Indeed, this is the case as both parameters $F F$ and $J_{\text {sc }}$ are nearly halved when no ODT is used. As listed in Table 1 a general correlation exists between flat-band voltage, fullerene cathode contact coverage, leakage current, and cell performance for cells comprising PCPDTBT:PC ${ }_{71} \mathrm{BM}$ as active layer. We highlight here that such correlation is also observed for P3HT, PCDTBT and 
PTB7-based cells blended either with $\mathrm{PC}_{61} \mathrm{BM}$ or $\mathrm{PC}_{71} \mathrm{BM}$ (see Table 1). For P3HT, PCDTBT annealing process increases the surface fullerene coverage (as deduced from $C-V$ analysis) in good agreement with the enhancement in $J_{\mathrm{sc}}$. 
Table 1: Device performance parameters of polymer:fullerene solar cells fabricated under different processing conditions.

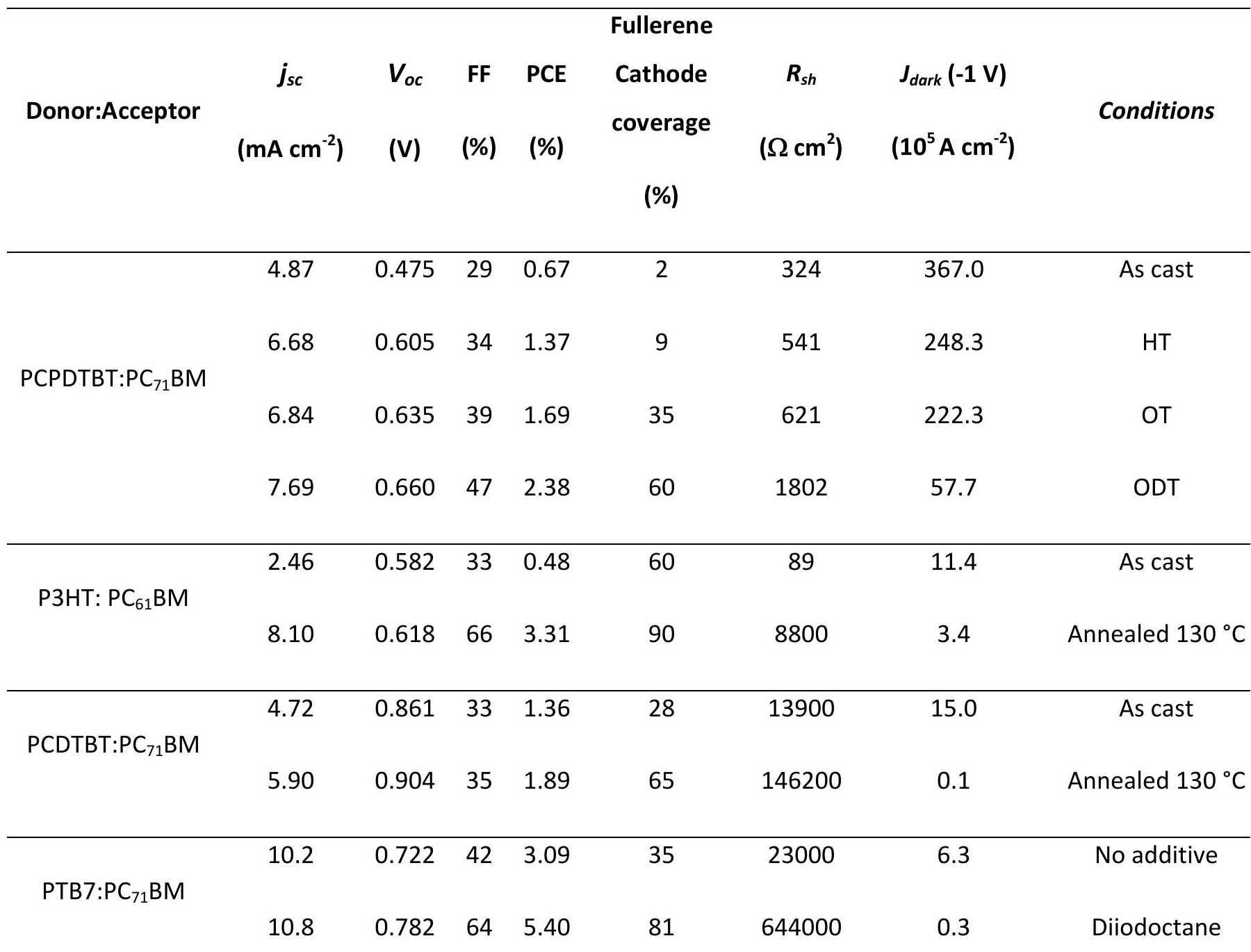



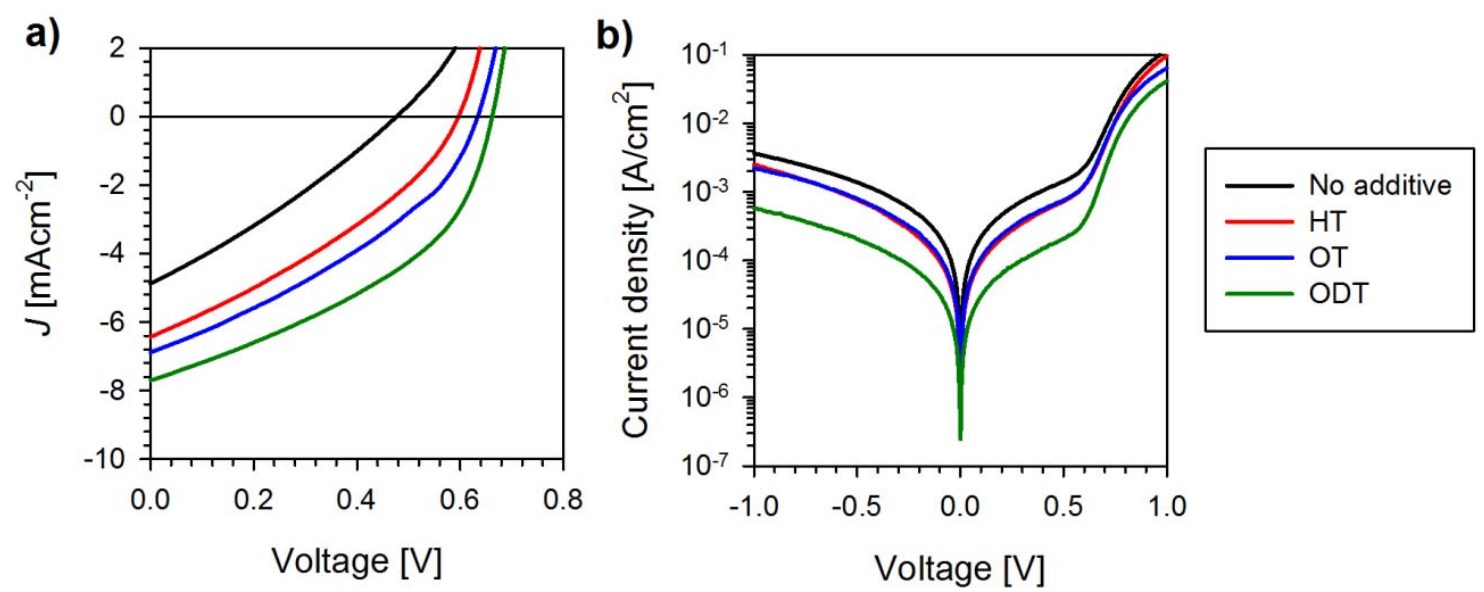

Figure 5: (A) $J-V$ response of BHJ soalr cells fabricated with the system PCPDTBT:PC ${ }_{71} \mathrm{BM}$ using different additives during processing: No additives, hexanethiol (HT), octanethiol (OT), octanedithiol (ODT). (B) Diode response of devices shown in (A) under dark conditions that shows different leakage current at negative bias.

\section{Conclusion}

We have demonstrated that a nanoscale-size fullerene-rich layer attaching the interface at cathode contacts induces higher electron selectivity, finally enhancing the device performance. It is shown how capacitance-voltage measurement is a sensitive technique to evaluate the relative donor/acceptor coverage at the blend/cathode interface. $C-V$ results are in good agreement with coverage values deduced from variable-angle spectroscopic ellipsometry. We have verified the validity of the technique with a variety of blends acting as active layers (PCPDTBT:PC ${ }_{71} \mathrm{BM}$, P3HT:PC 61 BM, PCDTBT:PC ${ }_{71} \mathrm{BM}$ and PTB7:PC $\left.\mathrm{PC}_{71} \mathrm{BM}\right)$. System-tailored thermal annealing and the use of proper additives are able to yield favorable vertical segregation profiles so as to induce fullerene-rich interfaces at the cathode.

\section{Methods}

Materials: PCPDTBT (1-Material), P3HT (Luminescece Tech.), PCDTBT (1-Material), PTB7(1-Material), $\mathrm{PC}_{61} \mathrm{BM}$ (Nano-C, $99 \%$ ), $\mathrm{PC}_{71} \mathrm{BM}$ (Nano-C, $99 \%$ ), hexanethiol (Alfa Aesar, $98 \%$ ), octanethiol (Alfa Aesar, $98 \%$ ), octanedithiol (Alfa Aesar, $98 \%$ ), diiodooctane (Alfa Aesar, 98 \%, copper stabilized), PEDOT:PSS (CLEVIOS P AI 
4083), chlorobenzene (Aldrich, $99.9 \%$ ), o-dichlorobenzene (Aldrich, $99.9 \%$ ), Ca (Aldrich, $99.995 \%$ ) and silver (Aldrich, $99.99 \%$ ) were used as received without further purification. All manipulations were carried out in a glovebox under a nitrogen atmosphere unless otherwise stated. PCPDTBT:PC ${ }_{71} \mathrm{BM}$ solutions (1:2 ratio) were prepared in $o$-dichlorobenzene $(17 \mathrm{mg} / \mathrm{ml})$ and were stirred at $60^{\circ} \mathrm{C}$ overnight before spin coating. Stock solutions of additives were prepared and were stirred for $30 \mathrm{~min}$ before addition to the solution containing PCPDTBT:PC ${ }_{71} \mathrm{BM}$. The solution that did not contain additives was diluted with additional $o$-dichlorobenzene to obtain the same final concentration. Once the additive and donor:acceptor solutions were mixed the mixtures were further stirred for 30 minutes at room temperature prior to device fabrication.

Preparation of a Vertically Segregated Case Study: The donor:acceptor system consisting of the low bandgap donor polymer PCPDTBT and $\mathrm{PC}_{71} \mathrm{BM}$ has previously been shown to provide differential phase segregation depending on the processing conditions. ${ }^{38}$ In particular, the use of the additive octanedithiol in different wt- $\%$ could totally modify the phase segregation as measured by VASE and GIXRD. In our study, we use the same donor:acceptor materials with a series of thiols with different alkyl chain lengths and/or number of thiol groups. Chlorobenzene solutions containing the following additives are used: hexanedithiol (HDT), octanethiol (OT) and Octanedithiol (ODT). Films of small active layer thicknesses $(40 \mathrm{~nm})$ were fabricated in the configuration substrate/active Layer with or without additives in order to measure profile concentration by VASE. The tested substrates were glass, glass/PEDOT and glass/ITO/PEDOT:PSS and similar trends were observed. The reported values here correspond to the latter. Reference films composed of pure $\mathrm{PC}_{71} \mathrm{BM}$ and pure PCPDTBT were also casted and measured. Additionally, selected active layer films were used in the configuration ITO/PEDOT:PSS/active layer for analysis using Kelvin Probe measurements. As discussed below, the information obtained from these two techniques is compared with that obtained from a set of devices (ITO/PEDOT:PSS/active layer/Ca/Ag) of the same active layer thickness. A second series of devices with $200 \mathrm{~nm}$ active layer thickness was also fabricated in order to test the validity of $C-V$ measurements under a different set of conditions. Due to the high molecular weight of PCPDTBT used in this work $(\sim \mathrm{Mw}=100 \mathrm{KDa})$ films were most homogeneous when the solution was added in the pre-rotating spin coater. This 
modification in the processing conditions by itself helps to explain the observed differences with previously reported donor:acceptor profiles for this system. Almost overlapping absorption spectra of neat polymer and blends with and without additives suggest that the degree of crystallinity of the polymer phase in the different samples do not differ greatly (see supporting information, Figure SI1).

Solar Cell fabrication: Devices with different donor/acceptor systems were also fabricated to test the general validity of the measurement and correlate donor/acceptor surface coverage with performance properties (see Supporting Information for details on preparation). In addition to PCPDTBT: $\mathrm{PC}_{71} \mathrm{BM}$-based solar cells, fabricated devices were based on P3HT:PCBM, PCDTBT:PC ${ }_{71} \mathrm{BM}$ and PTB7:PC ${ }_{71} \mathrm{BM}$ active layers. Polymer solar cells were fabricated with a standard sandwich structure of ITO/PEDOT:PSS/donor:acceptor/Ca/Ag, and $9 \mathrm{~mm}^{2}$ active area. PEDOT:PSS was spin coated in air at $5500 \mathrm{rpm}$ for 30 seconds onto an ITO coated glass substrate (10 $\mathrm{Ohm} / \mathrm{sq}$ ), resulting in a film thickness of $\sim 35 \mathrm{~nm}$. The substrates were heated at $120{ }^{\circ} \mathrm{C}$ for $10 \mathrm{~min}$ to remove traces of water and were transferred to a glovebox equipped with a thermal evaporator. PCPDTBT:PC $70 \mathrm{BM}$ layer was added onto the pre-rotating substrate at speeds of $1200 \mathrm{rpm}$ for 30 seconds at which stage the films are visually dried. However, traces of additives are expected to be completely removed during the subsequent processing steps. Sequential evaporation of calcium $(5 \mathrm{~nm})$ followed by a silver layer $(100 \mathrm{~nm})$ was carried out at a base pressure of $3 \times 10^{-6}$ mbar. Full devices, including top $\mathrm{Ca} / \mathrm{Ag}$ electrodes, were then encapsulated with epoxy and a glass slide before testing. Samples were characterized outside the glovebox.

Film and Device characterization: Variable angle spectroscopic ellipsometry (VASE) and photometry data were acquired at five angles between 55 and 75 using a Sopra GES-5E rotating polarizer spectroscopic ellipsometer (SEMILAB). The VASE data was modelled using the WinElli II piece of software. The Standard Critical Point model was employed accounting for the dielectric function of the pure materials. Several models were then tested in order to deduce the composition vertical profile of the blend films. These include continuous linear and parabolic profiles, as well as bilayer and trilayer effective structures. Overall, the trends observed for the different models in terms of the use of additives and surface coverage are consistent. The tri-layer 
model, however, was found to lead to the smallest fitting standard deviations, while providing good convergence of the fitting procedure and unique solutions for the deduced profiles when varying the initial conditions/parameters.

Kelvin Probe measurements were carried out with a KP010 system placed inside a glovebox from KP Technology equipped with a $2 \mathrm{~mm}$ wide tip. Films were fabricated in the glovebox and were measured without their exposure to air. Reference films for $\mathrm{PC}_{71} \mathrm{BM}$ and PCPDTBT were also fabricated for comparison. Kelvin Probe Force Microscopy (KPFM) measurements were performed with a scanning force microscope (Nanotec) equipped with Kelvin probe microscopy (KPM) capabilities. Freely available WSxM software was used for image acquisition and processing. ${ }^{39}$ Images were acquired working in non-contact dynamic mode using the oscillation amplitude as the feedback parameter. Platinum coated silicon tips (Budget Sensors), with nominal force constant of $3 \mathrm{~N} / \mathrm{m}$ and resonance frequency of $75 \mathrm{kHz}$ were used. Frequency modulation KPFM with an $\mathrm{AC}$ modulation bias of $500 \mathrm{mV}$ at $7 \mathrm{kHz}$ was used to measure the local contact potential. All the measurements were performed under a nitrogen atmosphere, however, samples were exposed to air between films were casted and measured. To be sure that the measurements were not affected by the proximity of the tip, the two trace method was used. During the first trace of a scan line, the Kelvin probe measurement was performed simultaneously with the topography scan. For the second pass, the Kelvin probe measurement was performed lifting up the tip 10-20 $\mathrm{nm}$ (following the topographic profile measured during the first pass). As reference sample for absolute values of work function was used HOPG (work function of $4.7 \mathrm{eV}$ ) measured between successive samples.

Current density-voltage characteristics were carried out under illumination with a $1.5 \mathrm{G}$ source $\left(1000 \mathrm{~W} \mathrm{~m}^{-2}\right)$ using an Abet Sun 2000 Solar Simulator. The light intensity was adjusted with a calibrated Si solar cell. Capacitance was recorded by applying a small voltage perturbation $(20 \mathrm{mV} \mathrm{rms})$ at $1000 \mathrm{~Hz}$. Measurements were carried out in the dark at several bias voltages to extract the capacitance-voltage characteristics. These measurements were performed with Autolab PGSTAT-30 equipped with a frequency analyzer module.

\section{Acknowledgement}

We thank financial support by Ministerio de Educacion y Ciencia (Spain) under 
project HOPE CSD2007-00007 (Consolider-Ingenio 2010), MAT2009-10642 and PLE2009-0086, and Generalitat Valenciana (Prometeo/2009/058, and ISIC/2012/008 Institute of Nanotechnologies for Clean Energies).

Supporting Information Available: Absorption, details on VASE and KPFM analyses, AFM images, surface coverage deduced from $C-V$ analysis for other polymer:fullerene blends, and details on preparation conditions. This material is available free of charge via the Internet at http://pubs.acs.org 


\section{References}

1.

Green, M. A.; Emery, K.; Hishikawa, Y.; Warta, W.; Dunlop, E. D., Solar Cell Efficiency Tables (Version 39). Prog Photovoltaics 2012, 20, 12-20.

2.

Yu, G.; Gao, J.; Hummelen, J. C.; Wudl, F.; Heeger, A. J., Polymer Photovoltaic Cells - Enhanced Efficiencies Via a Network of Internal DonorAcceptor Heterojunctions. Science 1995, 270, 1789-1791.

3.

Dennler, G.; Scharber, M. C.; Brabec, C. J., PolymerFullerene Bulk-Heterojunction Solar Cells. Adv. Mater. 2009, 21, 1323-1338.

4.

Servaites, J. D.; Ratner, M. A.; Marks, T. J., Organic Solar Cells: A New Look at Traditional Models. Energy Environ. Sci. 2011, 4, 4410-4422.

5.

Servaites, J. D.; Yeganeh, S.; Marks, T. J.; Ratner, M. A.,

Efficiency Enhancement in Organic Photovoltaic Cells: Consequences of Optimizing Series Resistance. Adv. Funct. Mater. 2010, 20, 97-104.

6.

Zhou, Y.; Fuentes-Hernandez, C.; Shim, J.; Meyer, J.;

Giordano, A. J.; Li, H.; Winget, P.; Papadopoulos, T.; Cheun, H.; Kim, J.; et al., A Universal Method to Produce Low-Work Function Electrodes for Organic Electronics. Science 2012, 336, 327-332.

7.

Steim, R.; Koglera, F. R.; Brabec, C. J., Interface Materials for Organic Solar Cells. J. Mater. Chem. 2010, 20, 2499-2512.

$8 . \quad$ Ratcliff, E. L.; Zacher, B.; Armstrong, N. R., Selective Interlayers and Contacts in Organic Photovoltaic Cells. J. Phys. Chem. Lett. 2011, 2, $1337-1350$.

9.

Po, R.; Carbonera, C.; Bernardia, A.; Camaioni, N., The Role of Buffer Layers in Polymer Solar Cells. Energy Environ. Sci. 2011, 4, 285-310.

10. Mishra, A.; Bäuerle, P., Small Molecule Organic Semiconductors on the Move: Promises for Future Solar Energy Technology. Angew. Chem.-Int. Edit. 2012, 51, 2020-67.

$11 . \quad$ Sun, Y.; Takacs, C. J.; Cowan, S. R.; Seo, J. H.; Gong, X.; Roy, A.; Heeger, A. J., Efficient, Air-Stable Bulk Heterojunction Polymer Solar Cells Using MoOx as the Anode Interfacial Layer. Adv. Mater. 2011, 23, 2226-2230.

12. Tseng, Y.-C.; Mane, A. U.; Elam, J. W.; Darling, S. B., Ultrathin Molybdenum Oxide Anode Buffer Layer for Organic Photovoltaic Cells Formed Using Atomic Layer Deposition. Sol. Energy Mater. Sol. Cells 2011, 99, 235239.

13.

Chen, W.; Nikiforov, M. P.; Darling, S. B., Morphology Characterization in Organic and Hybrid Solar Cells. Energy Environ. Sci. 2012, 5, 80458074.

14

Agostinelli, T.; Lilliu, S.; Labram, J. G.; Campoy-Quiles, M.; Hampton, M.; Pires, E.; Rawle, J.; Bikondoa, O.; Bradley, D. D. C.; Anthopoulos, T. D.; et al., Real-Time Investigation of Crystallization and Phase-Segregation Dynamics in P3HT:PCBM Solar Cells During Thermal Annealing. Adv. Funct. Mater. 2011, 21, 1701-1708.

15.

Chen, D.; Nakahara, A.; Wei, D.; Nordlund, D.; Russell, T. P., P3HT/PCBM Bulk Heterojunction Organic Photovoltaics: Correlating Efficiency and Morphology. Nano Letters 2010, 11, 561-567.

16. Zhu, S.; Liu, Y.; Rafailovich, M. H.; Sokolov, J.; Gersappe, D.; Winesett, D. A.; Ade, H., Confinement-Induced Miscibility in Polymer Blends. Nature 1999, 400, 49-51.

$17 . \quad$ Björström, C. M.; Nilsson, S.; Bernasik, A.; Budkowski, A.; Andersson, M.; Magnusson, K. O.; Moons, E., Vertical Phase Separation in SpinCoated Films of a Low Bandgap Polyfluorene/PCBM Blend-Effects of Specific Substrate Interaction. Appl. Surf. Sci. 2007, 253, 3906-3912.

18. Campoy-Quiles, M.; Ferenczi, T.; Agostinelli, T.; 
Etchegoin, P. G.; Kim, Y.; Anthopoulos, T. D.; Stavrinou, P. N.; Bradley, D. D. C.; Nelson, J., Morphology Evolution via Self-Organization and Lateral and Vertical Diffusion in Polymer:Fullerene Solar Cell Blends. Nat. Mater. 2008, 7, 158-164. 19. Xue, B.; Vaughan, B.; Poh, C.-H.; Burke, K. B.; Thomsen, L.; Stapleton, A.; Zhou, X.; Bryant, G. W.; Belcher, W.; Dastoor, P. C., Vertical Stratification and Interfacial Structure in P3HT:PCBM Organic Solar Cells. J. Phys. Chem. C 2010, 114, 15797-15805.

$20 . \quad$ Guerrero, A.; Marchesi, L. F.; Boix, P. P.; Ruiz-Raga, S.; Ripolles-Sanchis, T.; Garcia-Belmonte, G.; Bisquert, J., How the Charge-Neutrality Level of Interface States Controls Energy Level Alignment in Cathode Contacts of Organic Bulk-Heterojunction Solar Cells. ACS Nano 2012, 6, 3453-3460.

21. Bardeen, J., Surface States and Rectification at a Metal Semi-Conductor Contact. Phys. Rev. 1947, 71, 717-727.

22.

Vázquez, H.; Flores, F.; Oszwaldowski, R.; Ortega, J.; Pérez, R.; Kahn, A., Barrier Formation at Metal-Organic Interfaces: Dipole Formation and the Charge Neutrality Level. Appl. Surf. Sci. 2004, 234, 107-112.

23.

Vázquez, H.; Gao, W.; Flores, F.; Kahn, A., Energy Level Alignment at Organic Heterojunctions: Role of the Charge Neutrality Level. Phys. Rev. B 2005, 71, 041306(R).

24.

Burgués-Ceballos, I.; Campoy-Quiles, M.; Francesch, L.; Lacharmoise, P. D., Fast Annealing and Patterning of Polymer Solar Cells by Means of Vapor Printing. J. Polym. Sci. Pt. B-Polym. Phys. 2012, 50, 1245-1252.

25.

Engmann, S.; Turkovic, V.; Denner, P.; Hoppe, H.; Gobsch, G., Optical Order of the Polymer Phase within Polymer/Fullerene Blend Films. J. Polym. Sci. Pt. B-Polym. Phys. 2012, 50, 1363-1373.

26.

Germack, D. S.; Chan, C. K.; Kline, R. J.; Fischer, D. A.; Gundlach, D. J.; Toney, M. F.; Richter, L. J.; DeLongchamp, D. M., Interfacial Segregation in Polymer/Fullerene Blend Films for Photovoltaic Devices. Macromolecules 2010, 43, 3828-3836.

$27 . \quad$ Campoy-Quiles, M.; Nelson, J.; Bradley, D. D. C.; Etchegoin, P. G., Dimensionality of Electronic Excitations in Organic Semiconductors: A Dielectric Function Approach. Phys. Rev. B 2007, 76, 235206.

28

Kalinin, S. V.; Bonnell, D. A., Local Potential and Polarization Screening on Ferroelectric Surfaces. Phys. Rev. B 2001, 63, 125411.

$29 . \quad$ Cunningham, S.; Larkin, I. A.; Davis, J. H., Noncontact Scanning Probe Microscope Potentiometry of Surface Charge Patches: Origin and Interpretation of Time-Dependent Signals. Appl. Phys. Lett. 1998, 73, 123-125.

30 .

Peet, J.; Kim, J. Y.; Coates, N. E.; Ma, W. L.; Moses, D.; Heeger, A. J.; Bazan, G. C., Efficiency Enhancement in Low-Bandgap Polymer Solar Cells by Processing with Alkane Dithiols. Nat. Mater. 2007, 6, 497-500.

31. Boix, P. P.; Garcia-Belmonte, G.; Munecas, U.; Neophytou, M.; Waldauf, C.; Pacios, R., Determination of Gap Defect States in Organic Bulk Heterojunction Solar Cells from Capacitance Measurements. Appl. Phys. Lett. 2009, 95, 233302.

32. Bisquert, J.; Garcia-Belmonte, G., On Voltage, Photovoltage, and Photocurrent in Bulk Heterojunction Organic Solar Cells. J. Phys. Chem. Lett. 2011, 2, 1950-1964.

33. Ma, W.; Yang, C.; Gong, X.; Lee, K.; Heeger, A. J., Thermally Stable, Efficient Polymer Solar Cells with Nanoscale Control of the Interpenetrating Network Morphology. Adv. Funct. Mater. 2005, 15, 1617-1622.

34.

Blouin, N.; Michaud, A.; Leclerc, M., A Low-Bandgap Poly(2,7-Carbazole) Derivative for Use in High-Performance Solar Cells. Adv. Mater. 2007, 19, 2295-2300.

35.

Park, S. H.; Roy, A.; Beaupré, S.; Cho, S.; Coates, N.; Moon, J. S.; Moses, D.; Leclerc, M.; Lee, K.; Heeger, A. J., Bulk Heterojunction Solar Cells with Internal Quantum Efficiency Approaching 100\%. Nat. Photonics 2009, 3, $297-302$. 
36.

Alem, S.; Chu, T.-Y.; Tse, S. C.; Wakima, S.; Lu, J.; Movileanu, R.; Tao, Y.; Bélanger, F.; Désilets, D.; Beaupré, S.; et al., Effect of Mixed Solvents on PCDTBT:PC70BM Based Solar Cells. Org. Electron. 2011, 12, 1788-1793. $37 . \quad$ Liang, Y.; Xu, Z.; Xia, J.; Tsai, S.-T.; Wu, Y.; Li, G.; Ray, C.; Yu, L., For the Bright Future-Bulk Heterojunction Polymer Solar Cells with Power Conversion Efficiency of 7.4\%. Adv. Mater. 2010, 22, E135-E138.

38.

Agostinelli, T.; Ferenczi, T. A. M.; Pires, E.; Foster, S.; Maurano, A.; Müller, C.; Ballantyne, A.; Hampton, M.; Lilliu, S.; Campoy-Quiles, M.; et al., The Role of Alkane Dithiols in Controlling Polymer Crystallization in Small Band Gap Polymer:Fullerene Solar Cells. J. Polym. Sci. Pt. B-Polym. Phys. 2011, 49, $717-$ 724.

39.

Horcas, I.; Fernandez, R.; Gomez-Rodriguez, J. M.; Colchero, J.; Gomez-Herrero, J.; Baro, A. M., WSXM: A Software for Scanning Probe Microscopy and a Tool for Nanotechnology. Rev. Sci. Instrum. 2007, 78, 013705-8. 